\title{
EVALUATION OF THE GRAI INTEGRATED METHODOLOGY AND THE IMAGIM SUPPORTWARE
}

\author{
${ }^{1}$ Mr. J.M.C Reid \\ $\&$ \\ ${ }^{1}$ Prof. N.D. du Preez
}

\begin{abstract}
This paper describes the GRAI Integrated Methodology and identifies the need for computer tools to support enterprise modelling, design and integration. The IMAGIM tool is then evaluated in terms of its ability to support the GRAI Integrated Methodology. The GRAI Integrated Methodology is an Enterprise Integration methodology developed to support the design of CIM systems. The GRAI Integrated Methodology consists of the GRAI model and a structured approach. The latest addition to the methodology is the IMAGIM software tool developed by the GRAI research group for the specific purpose of supporting the methodology.
\end{abstract}

Keywords: Enterprise Integration, modelling, methodology, design, IDEF0, IMAGIM and GRAI.

\section{INTRODUCTION}

Work on the GRAI-GIM methodology started in the 1970's at the GRAI Laboratory of the University of Bordeaux. The objectives at the time, were to model a production management system in order to be able to define precisely the specifications needed to select a software package for a Computer Aided Production Management (CAPM) system. [G. Doumeingts et al, 1992]

With the subsequent developments of CIM (Computer Integrated Manufacturing) systems, the GRAI (Graphs with Results and Activities Interrelated) methodology was extended to include the design of the entire manufacturing system in order to support the design of such CIM systems. This was the start of GIM (GRAI Integrated Methodology). GRAI GIM, as it is now known, was developed as a methodology to support the design of CIM systems. By aiding the modelling of an Integrated Manufacturing System, the methodology provides the user with the specifications for the particular CIM system.

\footnotetext{
${ }^{1}$ Department of Industrial Engineering, Stellenbosch University
} 


\section{GRAI GIM AS AN EI METHODOLOGY}

GRAI GIM as developed by the GRAI Labouratory of the University of Bordeaux, resulted from production management studies initiated at the Labouratory as early as 1974. GRAI GIM is characterized by its use of the GRAI model, $d^{1}$ efining its use of the four co-operating systems (Decisional, Informational, Physical and Functional), and by the GRAI GIM structured approach ${ }^{1}$ emphasizing the life cycle of the CIM project. GRAI-GIM is intermediate between the CIM Open Systems Architecture (CIMOSA) and Purdue Enterprise Reference Architecture (PERA) in terms of the degree of formality implied and used and the consequent ease of understanding by the non-computer science educated user.

\subsection{The GRAI Model}

The GRAI Model, along with the structured approach forms the backbone of GIM. The model gives a graphic representation of a generic structure for the components of a CIM system along with the links between these components. The sub-systems that have been identified are as follows:

\section{Physical System}

The Physical sub-system constitutes all components of a CIM system such as machines, workers and techniques that are involved in the transformation of material and flow. This is similar to the resource systems described in other architectures.

\section{Decision System}

The Decision System represents the decision-making hierarchy within a CIM system. By splitting the decisional system according to hierarchical levels and then dividing the levels into functions, Decisional Centres can be identified. The identification and modelling of these decisional centers forms an important part of the GRAI Methodology. A decision center therefore represents decisions made within one function and at one hierarchical level.

\section{Information System}

The information system forms a link between the physical and the decisional system, as well as between these systems and their environment. This system is aimed at transforming and memorizing data.

\section{Functional View}

Within the GRAI model, the systems mentioned above namely the physical, decisional and informational systems, can be seen as three views. A view is defined as being a selective perspective from which a system is observed, concentrating on a particular aspect of that system and disregarding all other aspects and perspectives. A fourth view, namely the functional view, has been included in the GRAI Model. The role of the functional view is to create a simplified representation of the entire system. This simplified representation will show the main functions within the system as well as the interactions between these functions. The functional view therefore not only helps to create a simplified 
representation of the actual system, but also plays an important role in setting the boundaries of the study domain.

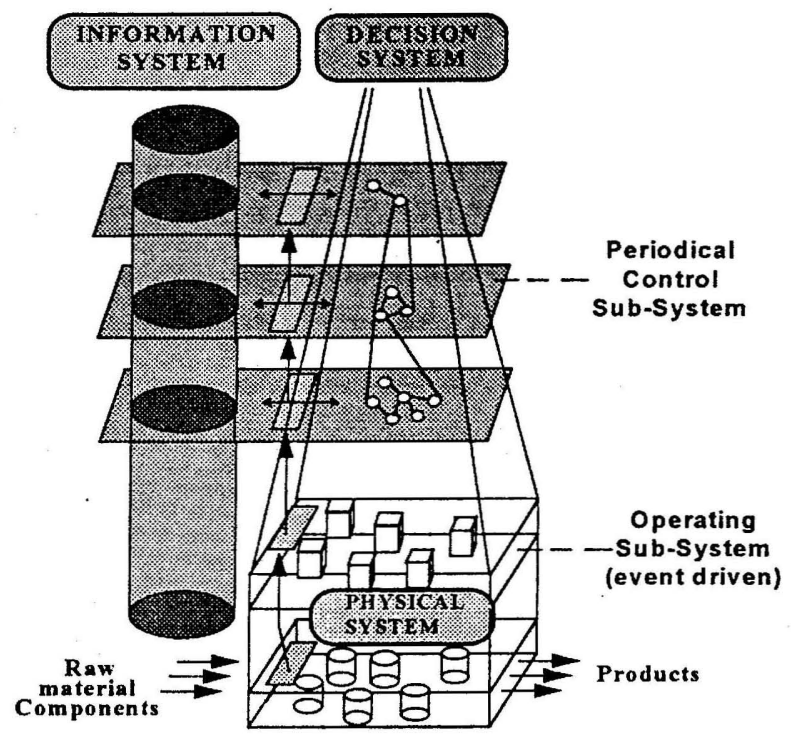

Source [G. Douneingts et al: GRAI Integrated Methodology A methodology for designing CIM systems]

Figure 1: The GRAI-GIM global model

\section{WHAT TOOLS ARE NEEDED}

Recent developments in the field of Enterprise Integration (EI) have all pointed towards the need for computer software tools to support the various methodologies. The magnitude of data that is involved in any EI project makes it impossible to perform such a project without the use of computers. The challenge that EI faces in the future, is the development of software support packages for the methodologies.

There are several commercially available modelling packages that are being used to aid EI projects around the world. These include the IDEF formalisms, Petri Nets, ARIS and Entity Relationship diagrams. Although these are powerful modeling formalisms, they have experienced limited success when used independently on integration projects. There is a definite need for customized packages that are able to support every aspect of the life cycle of an integration project. This level of support can not be achieved by simply modifying the existing formalisms. New modeling formalisms have to be developed which will satisfy the specific needs of a particular integration methodology.

The GRAI Methodology is the first of its kind to have gone this route. The GRAI research team identified the need to develop a software tool that is specific to 
GRAI GIM. This tool is known as IMAGIM and incorporates three formalisms that work together to form the GRAI model. The value of this tool lies in the ability to link the different models and in doing so facilitate integration.

The IMAGIM tool is however relatively immature in software terms, although commercial success has been achieved. It is hoped that this success will grow as the tool matures.

\section{THE IMAGIM TOOL}

IMAGIM is a computer tool that supports the GRAI Methodology. If used correctly, IMAGIM provides the analyst with a powerful graphic representation of the entity being integrated. The tool simplifies the GRAI modelling process and provides the analyst with the ability to create links or represent interactions between the models.

\subsection{Technical background to IMAGIM}

The IMAGIM software is being developed at Bordeaux University. IMAGIM was initiated within the context of the TIME-TOOL EUREKA project (Tools and methods for integration and for Management of Evolution of industrial firms), and has recently been commercialized by AUGRAI, the commercial leg of GIM. The software specifications were set up using OMT (Object Modelling Technique) object oriented analysis method, with the help of the software engineering tool PARADIGM Plus. The software is Windows based and all programming has been done in $\mathrm{C}++$.

\subsection{Components of IMAGIM}

The software tool consists of three modelling formalisms that are used to represent the four views in GRAI. The functional and physical models are represented by the IDEF0 (Icam DEFinition 0) formalism. The decisional system uses the GRAI Grids and the GRAI Nets and the informational system uses entity relationship diagrams.

\subsubsection{The IDEFO Formalism}

During the 1970's, the U.S. Air Force Program for Integrated Computer Aided Manufacturing (ICAM) sought to increase manufacturing productivity through systematic application of computer technology. During this program, the need for better analysis and communication techniques for people involved in improving manufacturing productivity was identified.

In order to satisfy this need, a series of formalisms known as the ICAM formalisms were developed. The IDEF0 formalisms were designed to facilitate the representation of functional models, where a functional model can be seen as a structured representation of the functions, activities or processes within the modeled system.

IDEF0 can be used in a wide variety of systems with varying degrees of automation. As a design tool, IDEF0 can be used to define the systems 
requirements and specify the necessary functions. In existing systems the formalism can be used to model and analyze the functions and provide a framework for the improvement of the system.

The IDEFO formalism provides the user with a model that consists of a hierarchical series of diagrams, text and glossary cross-referenced to each other. The two major components of the model are the functions, represented by blocks, and the data and objects that inter-relate the functions, represented by arrows.

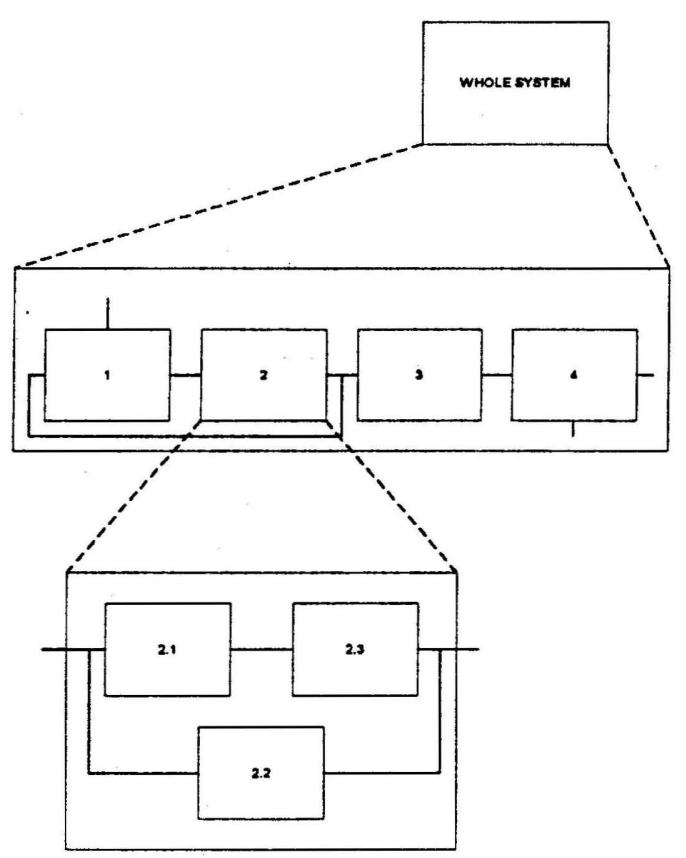

Figure 2:IDEF0 Model Structure

The diagram above (Figure 2:IDEF0 Model Structure) shows the hierarchical breakdown of the IDEF0 Model. It is this ability to follow a top down approach to modelling that makes this formalism suitable to support the GRAI Methodology.

\subsubsection{The GRAI Grids and Nets}

The GRAI grids and nets were developed by the GRAI group of LAP in Bordeaux. The grid serves as a map on which the decisional activities within a business entity can be mapped. The nets are then used to model the detailed decisional processes behind the grid. 


\subsubsection{The GRAI Grid}

The grid is a two dimensional map of the entity being model. The vertical axis represents the time frame within which a function has been performed, and the horisontal axis represents the type of function that is performed.

The time component of the decision was chosen because decisions are all valid for a certain period of time and these decisions are then reviewed after a certain time within this period. The time for which a decision is valid is called the horison of the decision, and the time after which the decision is reviewed is called the period. The notion of the period of a decision is closely related to the concept of control and adjustment. As a rule, the duration of a period should be one fifth of that of the horizon within which it falls. That means that the decision should be reviewed at least five times before the decision has been completed.

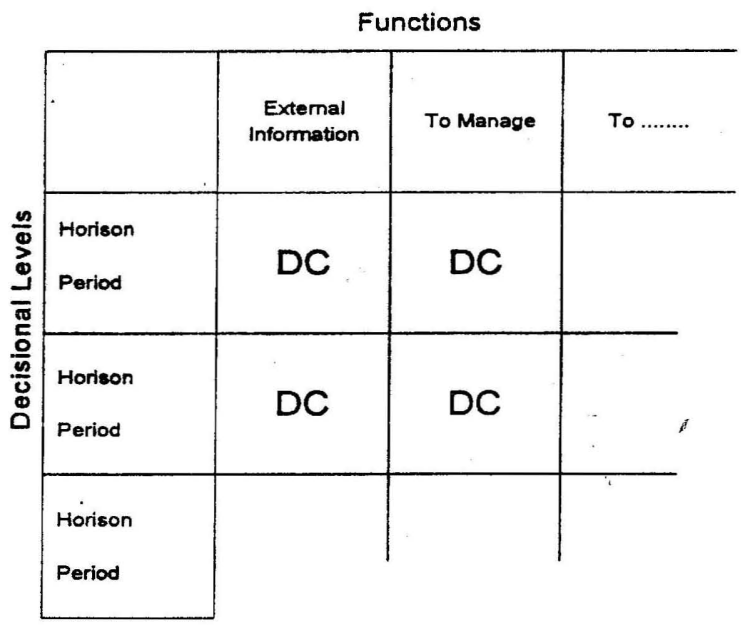

Figure 3: The Axes of the GRAI Grid

The functional component of the grid describes certain generic functions within the entity. There are two compulsory functions namely internal information and external information. The remaining functions are left to the user to decide, but it is important to remember that all relevant decisions need to be divided into these functions.

Once the functions and decisional levels have been established, the Decisional Centres (DC's) can be determined. DC's represent a decision or a group of decisions that are performed at a certain decisional level and within a certain function. The decisional levels, functions and DC's can be seen in Figure 3 above. It is important to note that not all of the potential DC's need to be populated.

Arrows in the grid represent the information from and interaction between the decisional centres, and between the decisional centres and the internal and external, 
environment. Single arrows represent information flow and double arrows represent the flow of decisional information i.e. information that is generated by a decision that is made.

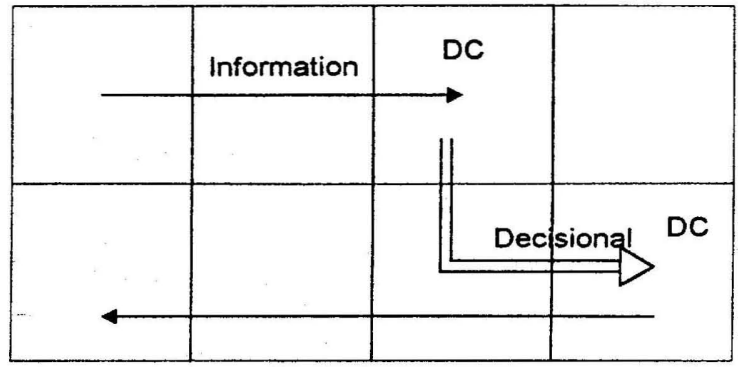

Figure 4: The Arrows used in the GRAI grid

The diagram above Figure 4 shows what the different arrows that are used on the grid look like. In the IMAGIM package the arrows are colour coded to enhance the visibility of the model.

The GRAI grids provide the user with an interesting model of the business entity. One of the first traps that new users fall into is the perception of time in the model. The only time elements in the grid are the horisons and periods. There is no calendar date or description of a specific point in time. The model represents ongoing functions that repeat themselves during the standard operation of the entity. The model is therefore a living one and should represent the present situation at any point in time.

\subsubsection{The GRAI Nets}

The nets were developed as a graphic tool for representing decisions made in the decisional centres. There are two types of nets, the first type are the more automated "to do" nets which represent decisions that may be made by simply following a set of rules. These decisions can typically be automated. The more advanced "to decide" nets rely heavily on the decision makers ability to make a decision using certain information, objectives, decisional variables, constraints and resources.

The GRAI nets give structure to the decisions made in the decision centres. They allow the user to document the decision making process and thereby capture information about decisions that are made. This information can be used to speed up the decision making process by eliminating the duplication of decisions and providing the decision maker with the necessary information regarding the objectives, decisional variables, constraints and resources that are available.

The real value of the nets in the IMAGIM tool lies in the ability to link the nets within and between decisional centres. The software tool allows the user to link certain nets to a decisional centre and then to link the DC's via the information 
flow between the nets. An example of this might be the use of information produced by a decision in one DC as a constraint or decisional variable for decisions in another DC.

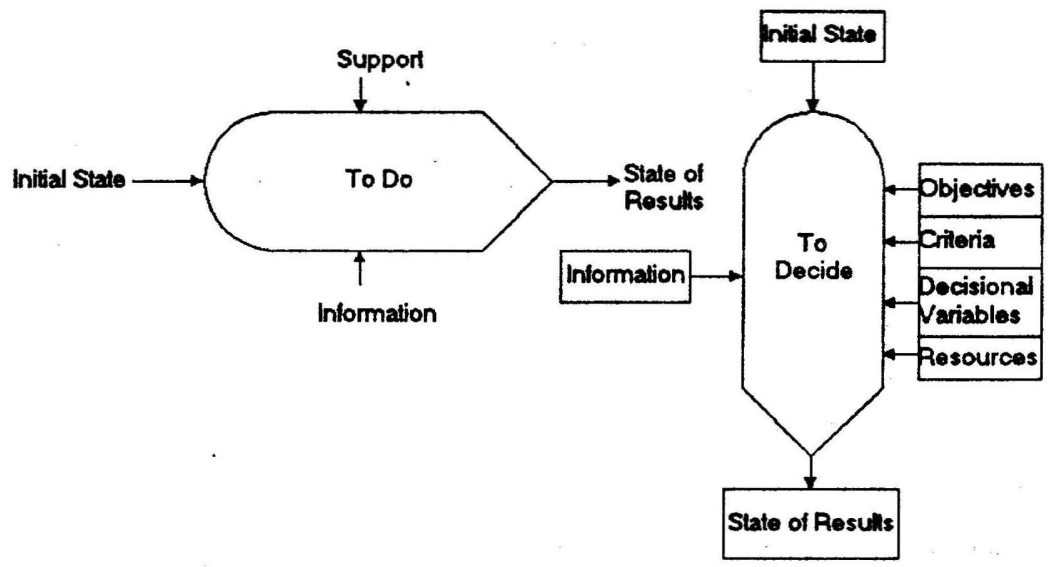

Figure 5: The GRAI Nets

\section{HOW IMAGIM SUPPORT GRAI GIM}

The IMAGIM software was developed to support the GRAI Methodology. The software is therefore very closely linked to the methodology. As has been mentioned, the Functional and Physical views are modeled using the IDEFo formalism. The IDEFO formalism is easy to model with and easy to understand. This is important because it means that users from a wide variety of fields are able to understand a single common model.

The Informational model is developed using entity relationship (ER) diagrams. ER diagrams are traditionally used in database specification and design. Once the ground rules of ER diagrams are understood, the ER diagrams are easy to interpret and provide valuable design specifications.

The most interesting module of the IMAGIM package is the module that defines the decisional view. The decisional view uses the grids and nets that were described in the previous section. The computerised grids and nets allow the user to gather and store large amounts of data about the decisional centres.

The real value of the IMAGIM package does not lie in any single formalism, but rather in the ability to create logical links between the formalisms. In this way, a certain process in the physical view can be linked to one or more decision in the decisional view. Similarly ER diagrams can be linked to decisional centres. The ability to interrelate the views adds value to the GRAI model as a whole. The IMAGIM package is therefore a valuable support tool for the GRAI-GIM methodology. 
There is however a lot of room for improvement. At present the tool's capabilities are limited to modelling. It is hoped that the IMAGIM tool will become more powerful in the future and include greater functionality through automatic consistency checking and linking between views. These and other aspects of IMAGIM are discussed in the remainder of this report.

\section{EVALUATION OF IMAGIM}

As has been mentioned IMAGIM does have its limitations. The tool is immature in software terms and requires added functionality and refinement. The tool has the potential to have intelligence built into it. By formulating a standard ontology and including this ontology in the programming of the software, the tool can be given the ability to perform consistency checks and automatic linking of the models. These improvements will open the door for more advanced functionality such as automatic model validation and report generation.

The IMAGIM tool also requires numerous cosmetic improvements to make it more user-friendly. These are listed below:

- Showing links between nets on the grid automatically would make modelling easier and help check the consistency.

- Storing information about the arrow that connects two decisional centres should be included.

- It should be possible to link nets from different decisional centres automatically.

- The help function for all the modules needs to be improved.

- Interaction of the GRAI models with other MS-windows ${ }^{\circledR}$ based programs such as word will help in the construction of reports and other documentation.

- The Methodology and software need to be expanded to include an Implementation Description.

- Changing the font sizes on the models in all the views has to be made more user-friendly.

- The drawing of arrows on the grids has to be made more flexible.

- The view area for the nets should be increased.

- The connecting arrows in the nets need to be easier to move.

Work is already under way to improve many of the aspects mentioned above. As has been mentioned IMAGIM is still young and will take time before it matures. It is however ahead in the field of customized EI methodology support tools and with sustained development will form a very powerful EI package along with the GRAI Methodology.

\section{CONCLUSIONS}

Enterprise Integration / Engineering is a growing discipline that will receive attention for many years to come. Due to the complexity of an integration project, 
it is necessary to use computers in enterprise modelling, design and implementation. There are a number of modelling tools which are commercially available, but these have not been tailored specifically for the existing EI methodologies and do not facilitate proper integration. The next generation of EI methodologies will be fully computer integrated and this requires the development of methodology specific software.

The GRAI group is one of the first research groups to develop software to support their methodology. This software, known as MMAGM, is developed around the GRAI-GIM global model, which consists of a functional, a physical, an informational and a decisional view. IMAGIM uses the IDEF0 formalism to model the functional and physical views and entity relationship diagrams for the informational view. The decisional view is modeled using GRAI grids and nets, which were developed along with the GRAI methodology.

IMAGIM is young in software development terms and although it has been commercially applied, further development is needed before it will be universally accepted. At present the GRAI group are working on improving the functionality of the program with the emphasis of consistency checking and automatic linking within the model. There are also several cosmetic changes necessary to improve the user-friendliness of the program. With sustained development IMAGIM and the GRAI methodology will contribute significantly in enterprise engineering applications in the future.

\section{REFERENCES}

1. Armistead C. \& Rowland P.; Managing Business Processes BPR and Beyond; John Wiley \& Sons.

2. Bernus P, Nemes L \& Williams J, Architectures for Enterprise Integration, First Edition 1996.

3. Doumeingts $\mathrm{G}$, Vallespir B, Zanetin $\mathrm{M}$, Chen D: GRAI INTEGRATED METHODOLOGY; A methodology for designing CIM systems, University Bordeaux 1 LAP/GRAI, version 1.0, 1992

4. Edosomwan J.A.; Organisational Transformation and Process Reengineering, The Quality Observer: St. Lucie Press Delray Beach, Florida.

5. Paulk, M.C. et al.; The Capability Maturity Model; IEEE Software, Vol. 10, No. 4, July 1993, pp. 18-27

6. Salvendy G: Handbook of Industrial Engineering, Second Edition, Institute of Industrial Engineering, 1991.

7. Scott Flaig, L; "Integrative Manufacturing: Transforming the organisation through people, process, and technology", 1993.

8. Vernadat F.B, Brandt D, Kosanke K, Nell J.G: Change in Organisation and Process Structures - Workshop 1, Working Group 3, Proceedings on . ICEIMT '97 International Conference on Enterprise Integration and Modeling Technology, Project 21.859.

9. Wolverton, J \& Cook, R; A Scorecard for Conducting a Small Business Performance Review, Internet: http://www.sbaer.uca.edu/docs/proceedings/94sbi330.txt 